\title{
HOW TO CONFIGURE YOUR QUANTUM DISAMBIGUATOR
}

\section{Follow these instructions carefully - your universe depends on it.}

\section{BY STEWART C BAKER}

${ }^{\mathrm{t}} \mathrm{p}$ t has come to our attention that a plurality of users has significant problems during the quantum disambiguator configuration process. These problems - many of which come from not pushing the red button located on the inside of your device - may include but are not limited to:

- Superposed instances of identical disambiguated worlds;

- Accidental creation of evil twins;

- Dead cats that are still alive (or vice versa);

- Sudden irrational activity that endangers the user's personal health as defined in a classical state (e.g. an avoidance of red buttons);

- Accidental auto-decapitation and/or persistent headaches;

- Visual hallucinations that suggest pushing the red button (if you have pushed the red button, please read the document titled So You've Accidentally Sentenced Every Sentient Being in the Known Universe to a Horrible and Instantaneous Death at your earliest convenience for instructions on how to revert to a pre-button world. If you have not pushed the red button, please do so at this time);

- Europe suddenly ceasing to exist, or being replaced by an improbably large banana;

- Sentient mathematical formulae which argue that the only way to really be safe from evil twins is to push the red button, no matter how compelling their evidence.

As a result of these and other problems, we would like to take this opportunity to provide our users with clear, straightforward instructions on how they may properly configure the quantum disambiguator to successfully untangle their hopelessly confused worlds.

1. Before beginning, wipe all currently disambiguated worlds from the disambiguator, being sure to ignore voices that encourage pushing the red button.

a) Push the red button.

2. Run your disambiguator through the default start-up procedure as outlined in the

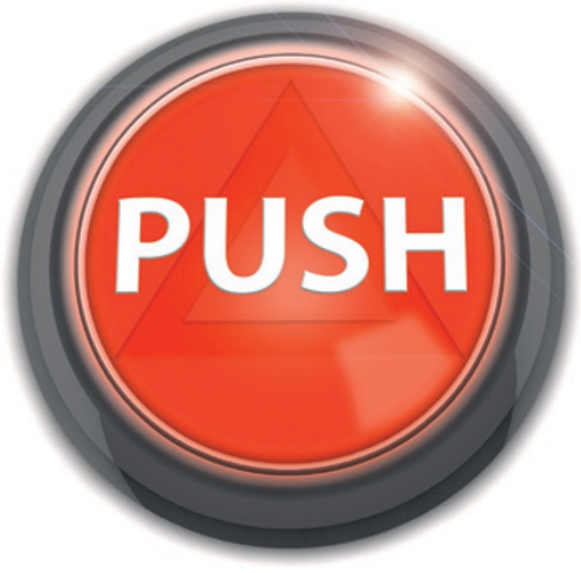

document titled World-Splitting Without the Headaches: Warming Up Your New Quantum Disambiguator.

a) If headaches persist, run through this step again, but wear a 5-star CRASH-rated helmet or duck a little earlier than you think is necessary.

3. Once you reach the configuration screen, use the following settings:

Collapse Threshold: $0.05 \mathrm{e}$

State Probability Threshold: 99\%

Bounding Conditions: Follow directions in document titled From Big Bang to Heat Death: Staying in Bounds with your Quantum Disambiguator

Evil Twin Goatee Style: Slick

Schrödinger Constant: Variable

Colour of Red Button: Blue

4. Hit 'Save'.

5 . Restart the disambiguator by pushing the blue button.

After running through these simple steps, almost all users report finding themselves in a world wherein their disambiguator is running without problems. Users who still have trouble, or who are unable to find one or more of the above configuration settings on the configuration screen, may wish to consider the very real possibility that they have entered an aberrant world-instance or been manipulated by an evil twin. These users may wish to read the appendix included at the end of this document or to call or e-mail $\rightarrow$ NATURE.COM

Follow Futures: @NatureFutures

f go.nature.com/mtoodm our help desk (hours vary until observed).

If, however, these steps do not resolve your problems, you may find yourself becoming increasingly frustrated. You may even consider pushing the red button, which is quite shiny and attractive and which you should probably just go ahead and push, as, statistically speaking, you've already pushed it in some other world and the worst has already happened.

No matter how frustrated you become, please do not push the red button. Doing so will set the number of potential observers in the Universe to zero, resulting in a new vacuum state across all possible worlds and causing instant death for all sentient beings, including the user. Note that if you have pushed the red button and are not yet dead, it is due to failsafes that have shunted you and your disambiguator to a pocket universe that will last just long enough for you to read the document mentioned at the beginning of this file and to regress to a pre-buttonpushing world.

In certain emergencies, collapse to a new vacuum state may seem desirable (e.g. if an evil twin is about to commandeer one's body through a nefarious and highly improbable string of events involving bananas and expertly timed visual hallucinations). Even in these cases, our development team suggests first waiting until the automated nightly recalibration in the hope that your twin will be noticed by our data-checking algorithms and returned to his or her own world.

Note that if your evil twin comes from a world in which the pressing of the red button has caused the Universe to collapse to a new vacuum state, he or she will experience a horrible and instantaneous death. This is not your fault, and any feelings of guilt should be assuaged by reading the pamphlet titled So You've Sentenced Your Evil Twin to a Horrible and Instantaneous Death. Please take care not to mistake this pamphlet for the similarly titled So You've Decided to Sentence Your Non-Evil Twin to a Horrible and Instantaneous Death - Again, unless you have first pushed the red button.

Push the red button. Please do not push the red button. Push the red button..

Stewart C Baker is an academic librarian, haikuist and speculative-fiction writer based in Oregon. His fiction has appeared in Daily Science Fiction, Flash Fiction Online and various other magazines. 